\title{
The statistical morphology of Saturn's equatorial ENA projections
}

Joe Kinrade ${ }^{1}$ Alex Bader ${ }^{1}$ Sarah Badman ${ }^{1}$ Chris Paranicas ${ }^{2}$ Don Mitchell ${ }^{2}$ Stan Cowley ${ }^{3}$ Gabby Provan ${ }^{3}$

\section{j.kinrade@lancaster.ac.uk}

Saturn is engulfed in a cloud of neutral gas that originates from ice fissures on the surface of Enceladus. Some particles collide and exchange charge, separating electrons and ions which are guided by Saturn's magnetic field. In this way, Saturn's rotating magnetosphere is loaded with mass, which eventually must be lost into space via ejections of plasma that stretch magnetic field lines to breaking point.

Some charged particles in the outer magnetosphere do not escape, but are fired back towards Saturn with field lines as they snap back into place. These energetic ions collide with neutrals, creating energetic neutral atoms (ENA) that were detectable using the INCA camera onboard Cassini. Pictures of Saturn's magnetosphere from INCA reveal dynamic regions of plasma flow, important for understanding the entire system.

We present an analysis of the INCA image set obtained throughout Cassini's mission. We've processed 670,000 images to characterise Saturn's average ENA morphology. Rings of ENAs are located at distances between 7-10 Rs, the point of peak interaction between the energetic ions and the neutral cloud. We also find ENA variation with Saturn's rotation period, associated with current systems that modulate the thickness of the plasmasheet every $\sim 10$ hours.

1 Physics Department, Lancaster University, Lancaster, UK

2 Johns Hopkins University Applied Physics Laboratory, Laurel, MD, USA

3 Department of Physics and Astronomy, University of Leicester, Leicester, UK

JGR Space Physics paper in preparation.

Also see data processing poster submission by Alex Bader. 


\section{Remotely sensing energetic neutral atoms with Cassini}

ENAs are produced at Saturn when an energetic ion collides with a neutral particle, and exchanges charge. The original ion becomes neutral but maintains its original energy and trajectory. No longer bound by the magnetic field, the new ENA whizzes off into space where we can detect it with the ion-neutral camera (INCA) onboard Cassini. Essentially, we're detecting the ENAs in much the same way as a camera sensor detects a photon. Because the ENA is a proxy for ion loss, if we're far enough away and looking in the right direction, we can take photographs of the global energetic ion activity throughout the entire magnetosphere.

By building up a picture of what the ENA distribution looks like, we can help to build on our global understanding of Saturn's magnetosphere and its plasma environment. One artist's impression of this is shown on the right, with an orange torus surrounding Saturn. This dense region of neutral gas spreads out throughout the magnetosphere, forming a sheet mostly confined by spin to the planet's equatorial plane making Saturn an efficient ENA emitter. From high latitude orbits, like the orientation shown in the upper right figure, we can project images onto the equatorial plane - or making an ENA map - for further analysis.

ENAs are useful, as they can tell us about both longterm plasma flow regions and the ring current behaviour, plus they react dynamically to transient events such as plasma injection from the magnetotail, or solar wind compressions.

\section{Physics

Cassini INCA

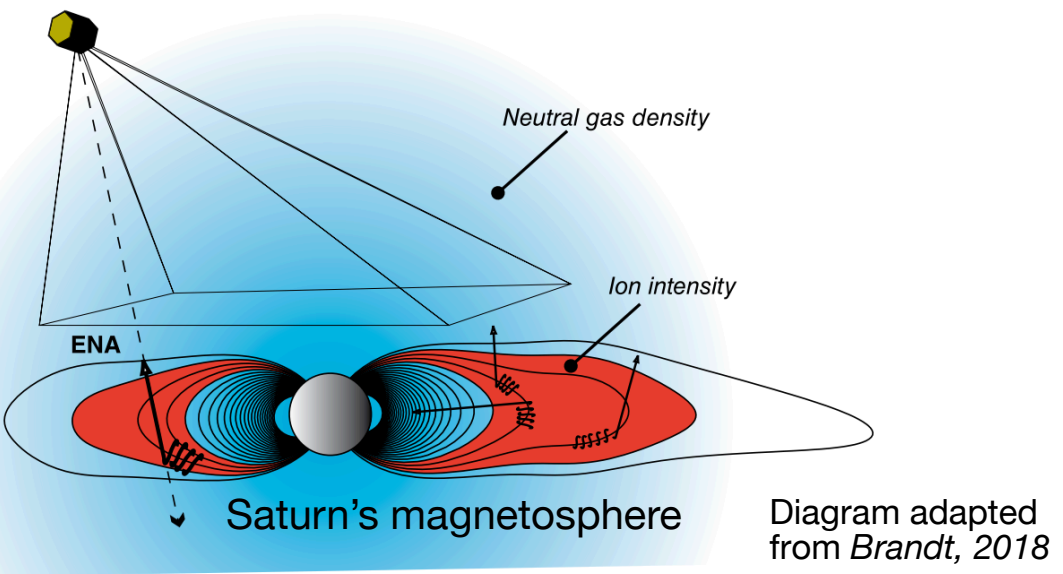

Artist's impression of Saturn's plasma environment based on 3 years of Cassini particle data. Krimigis +2007.

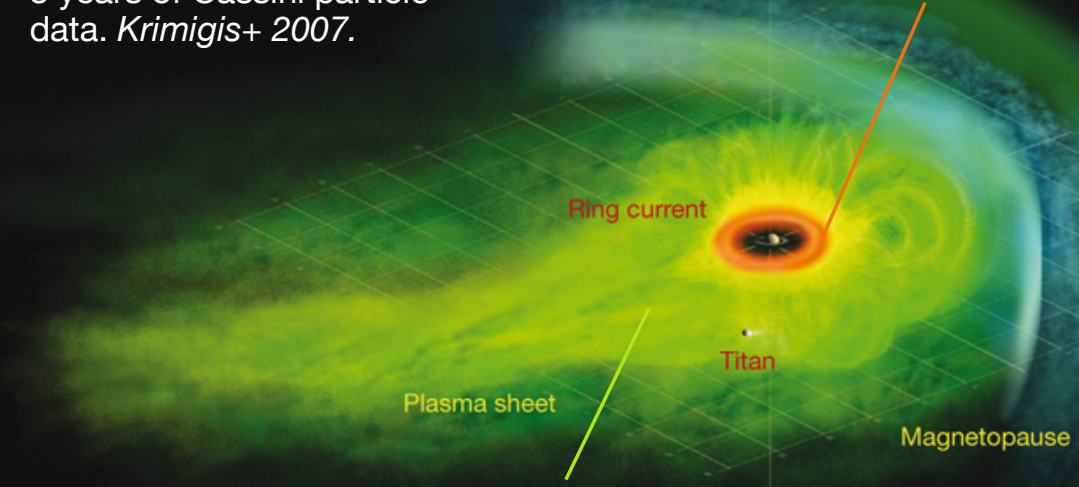

Energetic ions populate the plasma sheet, contributing to the global ring current
Tori of dense neutral gas outside the rings $\left(\mathrm{H}, \mathrm{O}, \mathrm{O}_{2}, \mathrm{OH}\right)$

Solar wind ב

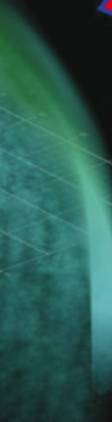




\section{Processing the entire INCA dataset}

Cassini's tour of Saturn lasted $\sim 14$ years, and during this time INCA took a lot of ENA images. Over 600,000! Each exposure is 4-5 minutes long, which adds up to $\sim 6$ years of accumulative exposure. An amazing achievement by the Cassini-INCA team.

But sifting through all the raw data is no small task. We've gone right back to the sensor count data, and have used an algorithm to sort, calibrate, and project all the imagery into a set of maps for analysis. We even had to use a supercomputer, which isn't as exciting as it sounds. An overview of the processing stages is shown on the right - you can learn more about the algorithm by viewing Alex Bader's poster entry. Lookout for a repository link to the ENA projection dataset as the JGR papers are released!

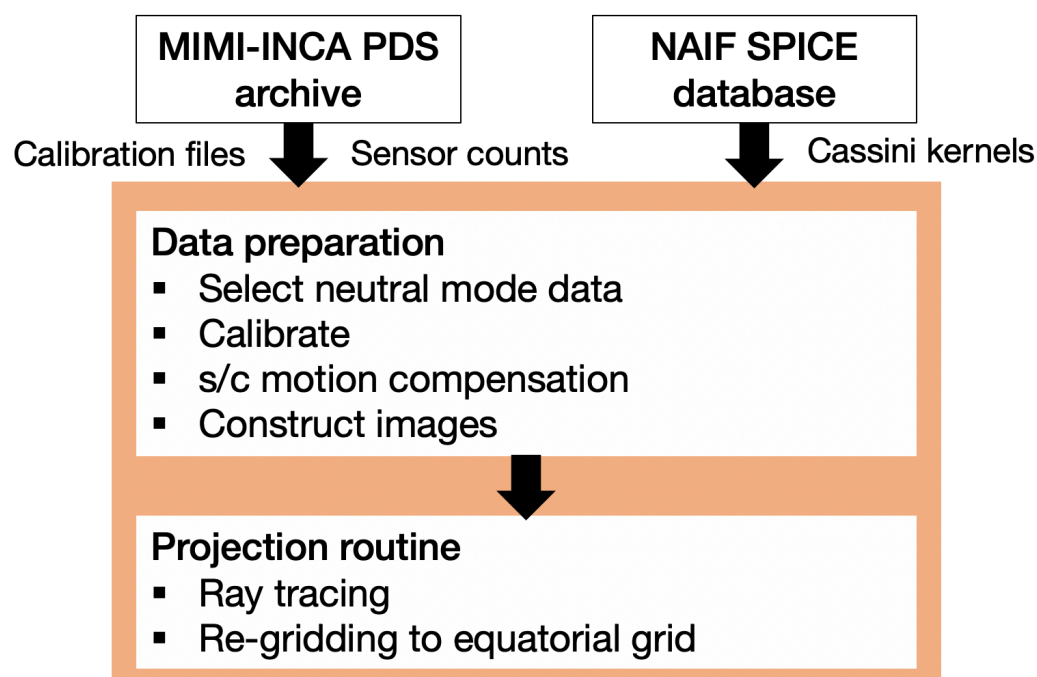

\section{Some ENA image processing steps:}

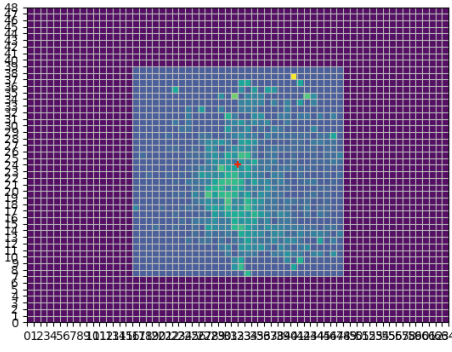

1. INCA sensor grid i.e. the raw photograph.
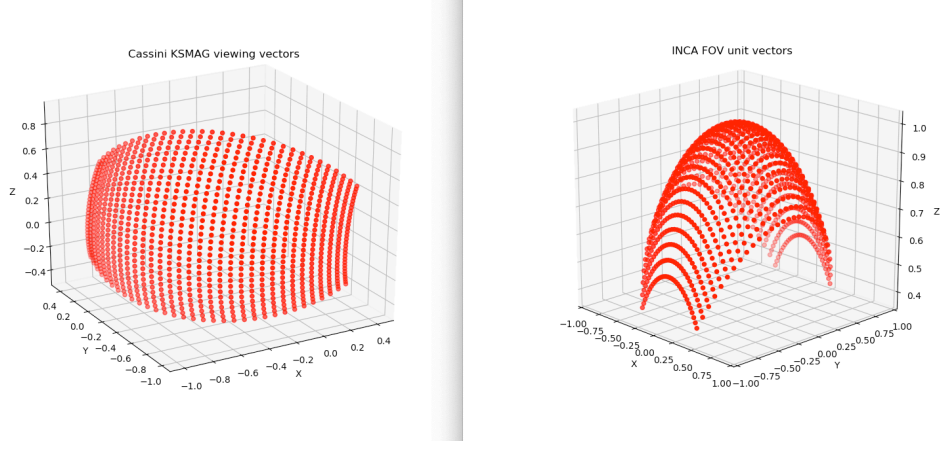

2. Coordinate transforms and spacecraft motion compensation.

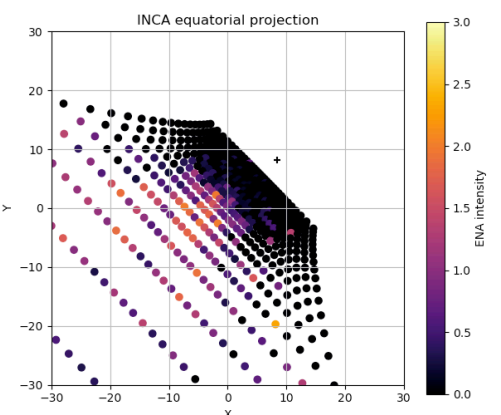

3. Line of sight pixel projection to the equatorial plane. 


\section{The statistical ENA morphology}

The long-time averaged morphology of the ENA emission forms a clear torus around Saturn. We sort of knew this before from some earlier studies, but this is the first time all the INCA data has been used to make this sort of ENA map. INCA was designed to detect ENAs originating from both hydrogen and oxygen ions, in low and high energy modes, so we have four separate images representing the Cassini-mission averages.

Because of the varying orbits that Cassini flew, we don't have uniform pixel coverage. You can see this in the map below, which shows the total high-latitude exposure time in each pixel after processing all those images. Some pixels have nearly 100 days of accumulative exposure, which is pretty good for a camera mounted on a spacecraft a billion km away. It's the most statistical picture of Saturn's ENAs we're going to get for quite a while...

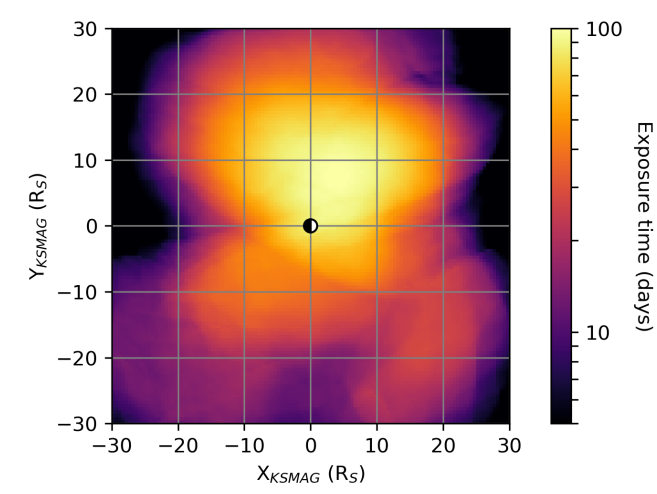

Total processed dataset exposure time $>50^{\circ}$ elevation

\section{Some potted observations:}

- The mean ENA intensities peak between $\sim 7-12$ RS. We can interpret this as the peak distance of ionneutral interaction. But remember this long-time average blurs out lots of the day-to-day dynamics that we see at Saturn.

- The ENA intensity is generally brighter on the nightside, where plasma injections are likely arriving from the magnetotail.

- The tori are offset toward the dayside by 1-2 $R_{S}$, possibly because of a global E-field asymmetry.
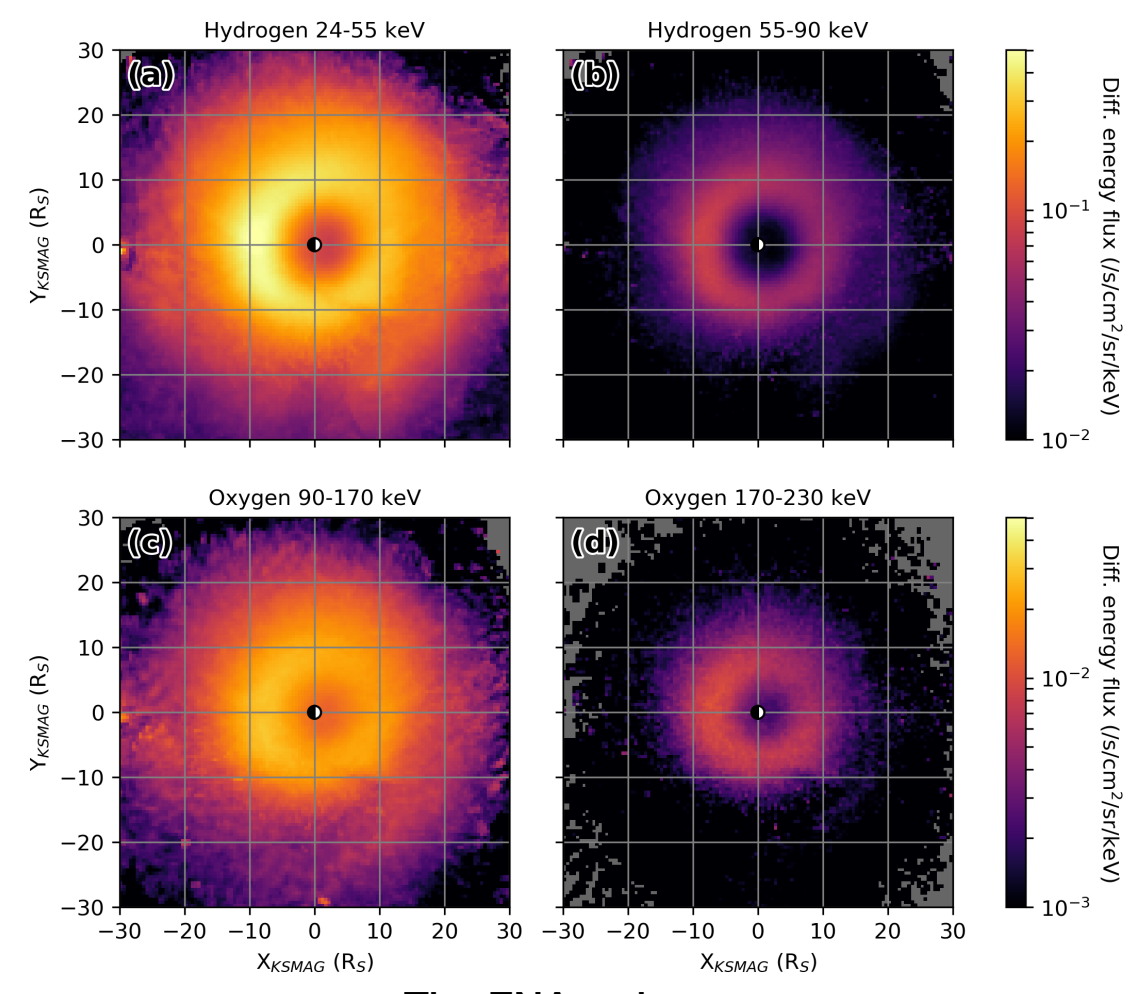

The ENA tori

What's next?

- How do the ENAs relate to Saturn's auroral emissions?

- We're seeing clear rotational modulation of the ENA intensity, related to Saturn's pesky rotating current systems and plasmasheet thickness variation. See upcoming paper for details.

- Track rotating ENA features to extract bulk plasma flow speeds, complimenting in-situ particle measurements. 